\title{
Application of a lateral intertubercular sulcus plate in the treatment of proximal humeral fractures: $A$ finite element analysis and example in clinical practice
}

\section{Dong Li}

Shandong University of Traditional Chinese Medicine

\section{WenXue LV}

Affiliated Hospital of Shandong University of Traditional Chinese

\section{WenMing Chen}

Affiliated Hospital of Shandong University of Traditional Chinese

\section{Jing Meng}

Shandong University of Traditional Chinese Medicine

\section{Song Liu}

Shandong University of Traditional Chinese Medicine

\section{ZongKang Duan}

Shandong University of Traditional Chinese Medicine

Bo Yu (D YBshengzhongyi@126.com)

Affiliated Hospital of Shandong University of Traditional Chinese

\section{Research Article}

Keywords: proximal humeral fracture, finite element analysis, lateral intertubercul arsulcus plate, medial support

Posted Date: April 26th, 2021

DOl: https://doi.org/10.21203/rs.3.rs-391928/v2

License: (c) (i) This work is licensed under a Creative Commons Attribution 4.0 International License. Read Full License 
4 Dong Li ${ }^{1, \dagger}$, WenXue Lv ${ }^{2, \dagger}$, WenMing Chen ${ }^{2, \dagger}$, Jing Meng ${ }^{1}$, Song Liu ${ }^{1}$, ZongKang Duan ${ }^{1}, \mathrm{Bo} \mathrm{Yu}^{*}{ }^{*}$

$5 \quad{ }^{1}$ Shandong University of Traditional Chinese Medicine, Jinan 250014, China.

$6{ }^{2}$ Department of Orthopedics, Affiliated Hospital of Shandong University of Traditional Chinese

7 Medicine, Jinan 250014, China

8 These authors contributed equally to this work.

$9 *$ Corresponding author

10 Bo Yu

11 Department of Orthopedics, Affiliated Hospital of Shandong University of Traditional Chinese

12 Medicine, Jingshi Road 16369, Jinan 250014, China

$13 \quad$ Tel: +86053168617087

14 E-mail: YBshengzhongyi@126.com

\section{Abstract}

Background: Inversion deformities caused by insufficient medial support are especially common

17 when the PHILOS locking plate is used to treat proximal humeral fractures. Using finite element analysis, the present study aimed to compare the biomechanical properties of a PHILOS locking plate (PLP) and a PLP combined with a lateral intertubercular sulcus plate (PLP-LSP) in the fixation of proximal humeral fractures with loss of the medial column. We also present the results of a 69-yearold female patient with a comminuted fracture of the proximal right humerus (Neer type four-part fracture) who underwent successful surgical treatment with a PHILOS plate combined with an auxiliary 
lateral intertubercular sulcus plate.

Methods: After creating a three-dimensional finite element model of a proximal humeral fracture with loss of the medial column, three implant models were established. A full-screw PLP was used in Group A, a PHILOS plate lacking medial screw support and an auxiliary plate (MPLP-LSP) was used in Group B, and a full-screw PLP-LSP was used in Group C. The three fixation models were applied to the proximal humeral fracture model, following which horizontal, compressive, and rotational loads were applied to the humerus model. We evaluated structural stiffness and stress distribution of the implant and compared displacement and angle changes among the three models.

Results: Displacement and angle changes were smallest in Group C (PLP-LSP) compared to those in Group A and Group B. The implant model used in Group C also showed the highest structural rigidity, endured less von Misses stress, and had the strongest stability than that used in Group A and Group

Conclusion: An LSP placed at the internodal grove not only aids in anatomical reduction but also provides effective lateral and medial support, thereby reducing stress on the PLP and providing better stability in patients with proximal humeral fractures.

Keywords: proximal humeral fracture; finite element analysis; lateral intertubercular sulcus plate; medial support

\section{Background}

Proximal humeral fractures are frequently encountered in clinical practice, with an incidence of $4 \%-5 \%$ 
currently include intramedullary nailing, internal fixation using a PHILOS locking plate (PLP), shoulder joint replacement, and other methods [2]. Although PLP is commonly used due to its wide scope of application [3], postoperative complications such as poor reduction, varus deformity, screw cutting, nonunion of fractures, infection, and limited function are frequently observed with its use [4] .Barlow et al. [5] followed up on 173 patients over 60 years of age with proximal humeral fractures who were treated with locking plate internal fixation. They reported failure rates of $26 \%, 39 \%$, and $45 \%$ for twopart (16 cases), three-part (23 cases), and four-part fractures (11 cases), respectively. Inversion deformities caused by insufficient medial support are especially common. Therefore, strengthening the medial support to reduce postoperative complications associated with the use of the PLP remains an urgent clinical need [6].

In clinical practice, several methods, such as allograft fibula implantation [7], titanium mesh implantation [8], bone cement [9], and auxiliary support plates [10-11], are used for strengthening the medial support. Authors have used a 1/3 tubular steel plate as an auxiliary plate to develop a shape according to the lateral anatomical structure of the internodal sulcus, placing the plate on the exterior of the internodal sulcus. During the operation, a Kirschner wire is first used for reduction, following which the auxiliary steel plate is inserted so that it can assist in the reduction according to the internodal groove. At the same time, the Kirschner wire can be removed to facilitate a multidirectional perspective. This surgical method has achieved good therapeutic effects, but whether the auxiliary steel plate can strengthen the medial support and enhance stability remains to be verified. Therefore, in the present study, we aimed to evaluate the biomechanical properties and stability of the auxiliary steel plate using finite element analysis.

66 
This study was approved by the Ethics Committee of the Affiliated Hospital of Shandong University of

Traditional Chinese Medicine, and all patients provided a written informed consent before participating in the study.

\section{Establishment of fracture model}

Standardized computed tomography (CT) data for the humerus were selected to establish a finite element model of the proximal humerus. CT data were obtained from a 27 -year-old healthy male.

The area of the simulated bone defect at the surgical neck of the humerus extended $5 \mathrm{~mm}$ laterally and $10 \mathrm{~mm}$ medially. We developed a three-dimensional model of the proximal humeral fracture to simulate instability of the medial column (Figure 1). The distinction between the cortical and cancellous bone was based on the gray measurement of the CT value, and the ranges of gray values for the cortical and cancellous bone were $662-1,841 \mathrm{HU}$ and 148-661 HU, respectively. The types of internals included the PLP and lateral auxiliary plate of the internodal groove. The PLP was $90 \mathrm{~mm}$ long and $3 \mathrm{~mm}$ thick with a screw length of $3.5 \mathrm{~mm}$. The auxiliary plate was $50 \mathrm{~mm}$ long and $2.5 \mathrm{~mm}$ thick with a screw length of $2.5 \mathrm{~mm}$. The arc of the plate was designed according to the anatomical structure of the exterior of the internodal groove.

\section{Implant assembly}

The PLP was assembled on the fracture model according to the standard operation method, and the upper end was $5 \mathrm{~mm}$ from the apex of the greater nodule. The auxiliary plate was tightly attached to the exterior of the intertubercular sulcus, and the upper end was $8 \mathrm{~mm}$ from the apex of the greater 
nodule. In one group, we used a full-screw PLP technique (PLP, Group A). In this group, the PLP was inserted with six locking screws at the proximal end and three locking screws at the distal end. In another group, we used MPLP-LSP (Group B). The MPLP-LSP was inserted with four screws at the proximal end while inserting the auxiliary plate. The two medial screw supports were not assembled. Two screws each were inserted at the proximal and distal ends of the auxiliary plate. In the third group, we used a full-screw PLP combined with an auxiliary plate (PLP-LSP, Group C). In this group, the PLPLSP was inserted with six locking screws at the proximal end and three locking screws at the distal while inserting an auxiliary plate with two screws at the proximal end and two screws at the distal end (Figure 2). The locking screw thread was omitted to simplify the model. The PLP model included 9,566 elements and 16,117 nodes. The auxiliary steel plate model included 1,781 elements and 3,314 nodes.

\section{Setting of parameters}

Finite element analysis was carried out using Abaqus 6.14 software (3DS, Waltham, MA). Linear elastic isotropic material properties were assigned to all models and placed materials. The elastic modulus of the normal cortical bone was set to $8,844 \mathrm{MPa}$, that of cancellous bone was set to $660 \mathrm{MPa}$, and that of the built-in steel plate was set to $114,000 \mathrm{MPa}$. The interface between the humeral head and glenoid was fixed in all models of the proximal humeral fracture. The contact behavior of the plate/locking-screw and bone/locking-screw interfaces was defined as fully fixed. The contact behavior of the plate/bone and cortical-screw/bone interfaces was defined as surface-to-surface. All contact elements were defined as deformable elements. The analyses were performed by assuming frictionless interactions to simplify the contact phenomena. Compression and rotation loads were applied to the humerus model to simulate the functions of the shoulder joint, including abduction, 
111 adduction, flexion, extension, axial compression, and internal and external rotation (Figure 3). Loads of $100 \mathrm{~N}$ were applied to the four directions of the humeral shaft to simulate the effects of shoulder

113 muscle abduction, adduction, flexion, and extension, and a load of $200 \mathrm{~N}$ was applied to the end of 114 the humerus to simulate axial compression. A torque of $7.5 \mathrm{Nm}$ was applied to the end of the humerus to simulate internal and external rotations [12].

\section{$117 \quad$ Evaluation indices}

\section{Fracture stability}

119 The vertical distance between points $c$ and $a b$ is defined as $e$, where $c, a$, and $b$ are the distal medial, proximal medial, and lateral points, respectively, of the fracture end, and $e$ is the displacement of the gap between the fracture ends. The stability of the fractured end was evaluated by measuring the change in the displacement (e) of the gap between the fracture ends (Figure 4).

\section{Rotational stability}

The rotational stability of the humeral head was evaluated by measuring the change in the angle ( $\alpha$ : the angle of the two straight lines; $a b$ and $c d$ ) between the proximal and distal fractures of the fractured end [13] (Figure 4). stress on the steel plate to evaluate the degree of stress. 


\section{Construct stiffness}

135 The compression and rotation stiffness values of the three models were calculated using finite element 136 analysis (Table 1). Compression and rotation stiffness values were $39.84 \mathrm{~N} / \mathrm{mm}$ and $110.20 \mathrm{Nm} / \mathrm{Rad}$ 137 in Group A (PLP), 43.67 N/mm and 153.50 Nm/Rad in Group B (MPLP-LSP), and 66.67 N/mm and 204.67 Nm/Rad in Group C (PLP-LSP), respectively.

\begin{tabular}{|c|c|c|}
\hline \multicolumn{3}{|c|}{ Table 1. Construct Stiffness } \\
\hline Group & Compression stiffness (N/mm) & Rotational stiffness (Nm/Rad) \\
\hline A & 39.84 & 110.20 \\
\hline B & 43.67 & 153.50 \\
\hline C & 66.67 & 204.67 \\
\hline
\end{tabular}

140 Group A (PLP): Full-screw PHILOS plate; Group B (MPLP-LSP): PHILOS plate without medial screw

141 support plus auxiliary plate; Group C (PLP-LSP): full-screw PHILOS plate plus auxiliary plate.

\section{Implant Stress}

144 The maximum equivalent stress and stress distribution of the three models were calculated using finite element analysis, as was the maximum von Mises stress for each model under different load conditions (Table 2). Three sets of model stress distributions and the maximum von Mises stress are shown in Figure 5.

In Group A, stress was concentrated near the support screw area during shoulder joint movement. 
suggesting that the auxiliary steel plate greatly disperses the stress, thereby reducing maximum stress.

151

\begin{tabular}{|c|c|c|c|c|c|}
\hline \multicolumn{7}{|c|}{ Table 2. Maximum von Misses Stress (MPa) } \\
\hline Group & Adduction & Abduction & Flexion & Extension & Axial compression \\
\hline A & 1,025 & 1,025 & 212.2 & 212.2 & 229.4 \\
\hline B & 892.6 & 892.6 & 283.8 & 283.8 & 198.6 \\
\hline C & 858.6 & 858.6 & 204.8 & 204.8 & 164.1 \\
\hline
\end{tabular}

Group A (PLP): Full-screw PHILOS plate; Group B (MPLP-LSP): PHILOS plate without medial screw

support plus auxiliary plate; Group C (PLP-LSP): full-screw PHILOS plate plus auxiliary plate.

Displacement changes

The displacement observed during different simulated activities for each model is displayed in Figure

6.

Angle changes

160 The angle changes measured during rotation were $3.9^{\circ}$ in Group A (PLP), $2.8^{\circ}$ in Group $B$, and $2.1^{\circ}$ in

161 Group C (Table 3)

\begin{tabular}{|c|c|c|c|}
\hline \multicolumn{4}{|c|}{ Table 3. Angle Changes during Rotation } \\
\hline Group & A & B & C \\
\hline Angle changes & $3.9^{\circ}$ & $2.8^{\circ}$ & $2.1^{\circ}$ \\
\hline
\end{tabular}


Till date, we have treated 14 patients with proximal humeral fractures using this surgical method and achieved good clinical treatment results. A typical case is described below. Figure 7 shows preoperative X-ray and computed tomography (CT) findings for a 69-year-old female patient with a comminuted fracture of the proximal right humerus (Neer type four-part fracture). The patient underwent surgical treatment with a PLP-LSP. The five-hole, one-third tubular steel plate was shaped according to the external anatomical structure of the internodal sulcus and attached to the exterior of the internodal sulcus (see Figure 8). Re-examination after 3 months revealed a healed fracture, and the patient exhibited good functional recovery (see Figure 9).

\section{Surgical technique in clinical practice}

174 After general anesthesia or brachial plexus anesthesia, the patient was placed in the supine position at shoulder height. Routine disinfection and draping of the surgical area were performed, following which an anterior medial incision of approximately $10 \mathrm{~cm}$ in length was made. The skin, subcutaneous tissue, and fascia were cut sequentially and separated from the pectoralis major and deltoid muscles. tissue displacement and blood clotting, Kirschner wire was used to pry the humeral head to restore the neck-stem angle and temporarily reset and fix the broken end to expose the intermuscular groove of the biceps. After pre-bending and shaping, the auxiliary steel plate was attached to the internodules to guide the reduction on the lateral side of the groove. The angle was adjusted during the bone plate is not hindered, the five-hole $1 / 3$ tubular steel plate is sufficient. The top of the plate was 

part of the fracture, and allogeneic bone was appropriately implanted according to the fracture defect. After confirming a correct positioning of the screw plate under fluoroscopy, the rotator cuff was sutured with a tendon suture. The incision was flushed, sutured, and wrapped in a sterile dressing, and a drainage tube was placed at the incision.

\section{Discussion}

In the present study, we used finite element analysis to explore the biomechanical properties of a lateral plate at the intertubercular groove in a model of proximal humeral fracture with loss of the medial column. Our findings indicated that a higher structural stiffness under axial compression and rotational load was associated with a stronger ability of the internal fixation system to prevent varus displacement of the humeral head. Our comparison between Groups A and B indicated that the auxiliary steel plate can completely replace the support function of the supporting screws while ensuring greater structural rigidity. Results from Group C further suggest that combining the auxiliary plate with the original PLP leads to an even greater structural rigidity and stability than that observed in Groups A and B while improving the bone defect area. Regardless of the force applied in the horizontal, vertical, and torsional directions, the changes in displacement and angle in Group C were only one half of those observed in Group A, indicating that the steel plate significantly increases the stability and medial support of the original PHILOS system. In addition, the maximum von Mises stress on the internal fixation can reflect the load transfer methods of different internal fixation methods, and the higher the von Mises stress, the greater the torsional force .Thus, after a long period of repeated twisting, the internal fixation is the part most likely to fail. The maximum von Mises stress 
values were smallest in Group C under various loads compared to those of the other groups. Therefore, these results indicate that the auxiliary steel plate can provide a better internal support, effectively disperse stress, reduce the risk of internal fixation failure, and enhance the stability of the internal

211 fixation.

Screw cutting and varus displacement of the humeral head are the most common surgical complications of open reduction and internal fixation of proximal humeral fractures. Lack of a medial support has been cited an important reason for postoperative complications and surgical failure [14], and the two medial support screws of the PLP plate are particularly important for ensuring medial support of the proximal humerus [15,16]. A comparative study by Shen et al. [17] reported that placement of the medial support screw greatly reduced screw cutting, varus deformity, and the probability of a secondary surgery. In clinical practice, various methods such as autologous or allogeneic fibula implantation, titanium mesh implantation, and the use of an auxiliary steel plate are used to compensate for the effect of the medial support screw and strengthen medial support [7-11]. doctors have high technical experience. In addition, the cost and risk of infection and disease transmission are high, and the supply is limited [18]. Although an auxiliary plate placed on the inner side of the proximal humerus can directly provide an effective medial support, the medial approach is not easy to learn due to the complex anatomy of the neurovascular structure. Improper techniques can easily lead to iatrogenic nerve and blood vessel damages, which explain why the medial plate approach for proximal humeral fractures has not been clinically promoted. With our technique, the auxiliary steel plate is pre-bent (1/3 tubular steel plate) according to the anatomical shape of the exterior of the internodal sulcus. This is because the internodal sulcus can be used as a landmark to 
assist anatomical reduction [19], and the lateral side of the internodal sulcus can be easily exposed

without additional trauma during the operation. The conventional anteromedial approach can reduce

232 the risk of damaging muscle nerve branches [20]. After the auxiliary steel plate is placed for temporary fixation during the operation, the Kirschner wire used to maintain the reduction can be removed, which is convenient for multi-angle fluoroscopy and shortens the operation time. the application of finite element analysis in orthopedic biomechanics has evolved, and it is widely used to evaluate new implants or materials, strain and stress distribution, and load transfer between objects and bones [22]. However, given the complex structure of the shoulder joint, it is impossible to accurately simulate the real boundary conditions of the interaction of all muscles and ligaments.

Our research aims to simplify the study of the shoulder joint by ignoring the interactions of muscles, ligaments, bones, and other surrounding structures [23]. Although finite element analysis can simulate the properties of various bone materials and load forces in various directions, it does not fully reflect the real-world situation due to differences in bone density and fracture types among patients.

\section{Conclusion}

In summary, our findings demonstrate that an LSP placed at the internodal groove not only aids in anatomical reduction but also provides effective lateral and medial support, thereby reducing stress on the PLP plate and providing better stability in patients with proximal humeral fractures. Moreover, this placement allows for easy exposure, which reduces additional trauma and blood loss. In addition, the plate can be reset according to the internodal sulcus to facilitate fluoroscopy. Given that this technique may also reduce the risk of complications and increase the stability of the internal fixation, 

fractures.

\section{List of abbreviations}

256

CT: computed tomography; PLP: full-screw PHILOS locking plate; MPLP-LSP: PHILOS plate lacking medial screw support plus auxiliary plate; PLP-LSP: full-screw PHILOS locking plate plus auxiliary plate

\section{Declarations}

The authors declare that the research was conducted in the absence of any commercial or financial relationships that could be construed as a potential conflict of interest.

Availability of data and materials

The datasets used and/or analyzed during the current study are available from the corresponding author on reasonable request.

\section{Ethics approval and consent to participate}

This study was performed in accordance with the ethical standards of the Institutional Ethics accordance with the 1964 Declaration of Helsinki and its later amendments or comparable ethical standards. The participant provided written consent to participate in our study.

Consent for publication

271 Written consent for publication was obtained from the participant described in this article.

273 The authors declare that they have no competing interests. 
275 This work was financially supported by grants from the Clinical Medical Science and Technology 276 Innovation Program of the Jinan Science and Technology Bureau (202019156), Traditional Chinese 277 Medicine Technology Development Plan of Shandong Province (2019-0154), and Natural Science 278 Foundation of Shandong Province (ZR2016HM43).

\section{Authors' contributions}

280 Bo Yu and WenXue Lv contributed to the conception and design, performance of the experiments, data analysis, and interpretation; Dong Li and WenMing Chen performed the data analysis and manuscript writing; Jing Meng, Song Liu, and ZongKang Duan contributed to the performance of the experiments and data analysis. Bo Yu contributed to the conception and design, financial support, data analysis and interpretation, manuscript writing, and final approval of the manuscript. All authors read and approved the final manuscript.

Acknowledgments

We would like to thank Editage (www.editage.cn) for English language editing.

\section{References}

1. Bell JE, Leung BC, Spratt KF, Koval KJ, Weinstein JD, Goodman DC, et al. Trends and variation in incidence, surgical treatment, and repeat surgery of proximal humeral fractures in the elderly. J Bone Joint Surg Am. 2011;93:121-31.

2. Mease SJ, Kraeutler MJ, Gonzales-Luna DC, Gregory JM, Gardner MJ, Choo AM. Current controversies in the treatment of geriatric proximal humeral fractures. J Bone Joint Surg Am. 2021; doi:10.2106/jbjs.20.00665. 

2007 to 2016. Arch Orthop Trauma Surg. 2019;139:1713-21. affecting proximal humerus fixation stability. J Orthop Trauma. 2021;35:259-64.

5. Barlow JD, Logli AL, Steinmann SP, Sems SA, Cross WW, Yuan BJ, et al. Locking plate fixation of proximal humerus fractures in patients older than 60 years continues to be associated with a high complication rate. J Shoulder Elbow Surg. 2020;29:1689-94.

6.Oppebøen S, Wikerøy AKB, Fuglesang HFS, Dolatowski FC, Randsborg PH. Calcar screws and adequate reduction reduced the risk of fixation failure in proximal humeral fractures treated with a locking plate: 190 patients followed for a mean of 3 years. J Orthop Surg Res. 2018 Aug 9;13(1):197.

7. Kim YK, Kang SW, Jung KH, Oh YK. The potential of locking plate with intramedullary fibular allograft to manage proximal humeral fracture with an unstable medial column. Arch Orthop Trauma Surg. 2020.

8. Della Rotonda G, Guastafierro A, Viglione S, Russo F, Coscione AV, Ciccarelli M, et al. Analysis of early and late clinical and radiologic complications of proximal humeral fractures using open reduction, internal fixation, and intramedullary titanium cage augmentation. J Shoulder Elbow Surg. 2020;29:1843-51.

9. Kim DY, Kim TY, Hwang JT. PHILOS plate fixation with polymethyl methacrylate cement augmentation of an osteoporotic proximal humerus fracture. Clin Shoulder Elb. 2020;23:156-8. humerus fractures. Injury. 2014;45:1280-2. 
short-term clinical efficacy for the treatment of proximal humerus fractures with the combined use of medial anatomical locking plate fixation and minimally invasive lateral locking plate fixation. J Orthop Surg Res. 2021;16:29.

12. He Y, He J, Wang F, Zhou D, Wang Y, Wang B, et al. Application of additional medial plate in treatment of proximal humeral fractures with unstable medial column: A finite element study and clinical practice. Medicine (Baltimore). 2015;94:e1775. method for proximal humeral fractures without medial support. J Orthop Surg Res. 2017;12:72. in locked plating of proximal humerus fractures. J Orthop Trauma. 2007;21:185-91. 15. Yang P, Zhang Y, Liu J, Xiao J, Ma LM, Zhu CR. Biomechanical effect of medial cortical support and medial screw support on locking plate fixation in proximal humeral fractures with a medial gap: A finite element analysis. Acta Orthop Traumatol Turc. 2015;49:203-9. the maintenance of fracture reduction after locked plating of proximal humerus fractures. Chin Med J (Engl). 2018;131:1827-33.

17. Shen P, Zhu Y, Zhu L, Li X, Xu Y. Effects of medial support screws on locking plating of proximal humerus fractures in elderly patients: a retrospective study. Ann Transl Med. 2019;7:560. 18. Gardner MJ, Boraiah S, Helfet DL, Lorich DG. Indirect medial reduction and strut support of proximal humerus fractures using an endosteal implant. J Orthop Trauma. 2008;22:195-200. intertubercular sulcus and retroversion angle of humeral head. Int J Clin Exp Med. 2015;8:4837-45. 

approaches to the proximal humerus: A quantitative comparison of the deltopectoral approach and

342 the anterolateral acromial approach. J Am Acad Orthop Surg Glob Res Rev. 2018;2:e017.

21. Brekelmans WA, Poort HW, Slooff TJ. A new method to analyse the mechanical behaviour of skeletal parts. Acta Orthop Scand. 1972;43:301-17.

22. Ye Y, You W, Zhu W, Cui J, Chen K, Wang D. The applications of finite element analysis in proximal humeral fractures. Comput Math Methods Med. 2017;2017:4879836.

23. Büchler P, Farron A. Benefits of an anatomical reconstruction of the humeral head during shoulder arthroplasty: a finite element analysis. Clin Biomech (Bristol, Avon). 2004;19:16-23.

\section{Figure Legends}

Figure 1. Bone defect area. A bone defect area with a width of $5 \mathrm{~mm}$ on the lateral side and $10 \mathrm{~mm}$ with an unstable medial column.

Figure 2. Three implant models. A: PHILOS plate (PLP). B: PHILOS plate lacking medial screw support plate (PLP-LSP). 


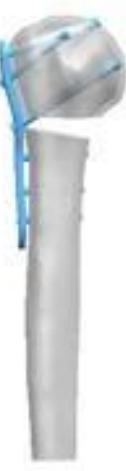

358

359

360

361

362

363

364

365

366 (angle $\alpha$ ).

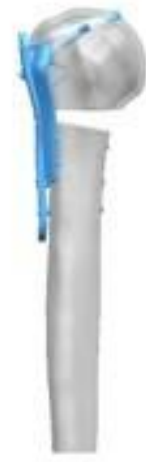

B

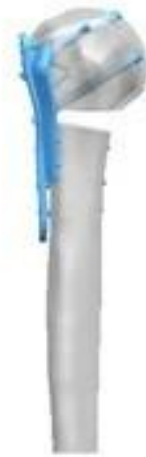

C

Figure 3. Load application. Compressive and rotational loads were applied to the humerus model to simulate the functions of the shoulder joint, including abduction, adduction, flexion, extension, axial compression, and internal and external rotation.
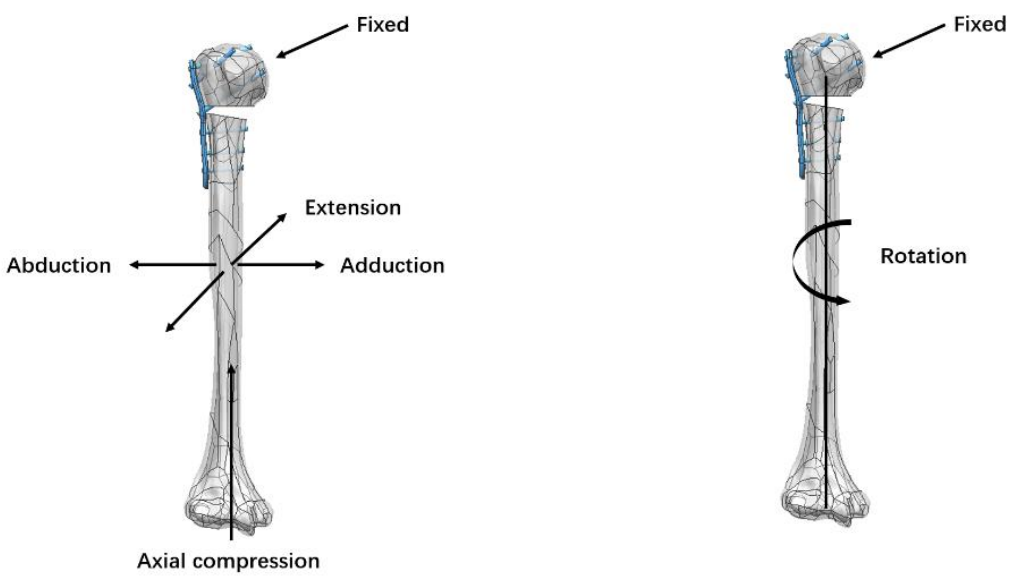

Figure 4. Stability. The stability of the fracture region under horizontal and compressive loads was assessed based on the distance covered by the medial fracture gap (line e). The angular variation between the proximal and distal fracture gap was determined to assess regional rotational stability 

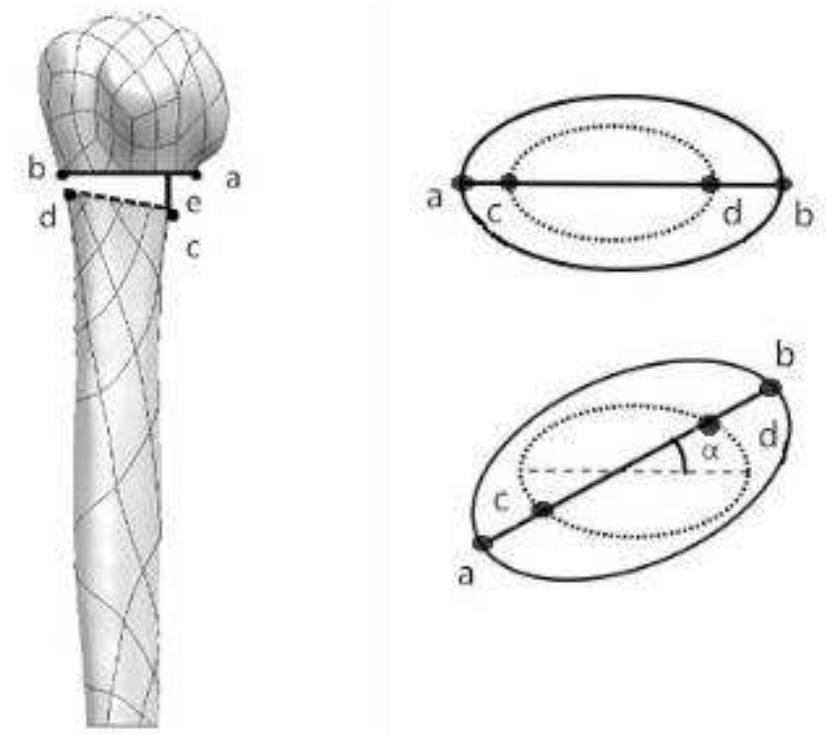

368 Figure 5. The maximum von Misses stress and stress distribution. Group A (PLP): Full-screw 369 PHILOS plate; Group B (MPLP-LSP): PHILOS plate without medial screw support plus auxiliary plate;

370 Group C (PLP-LSP): full-screw PHILOS plate plus auxiliary plate. 

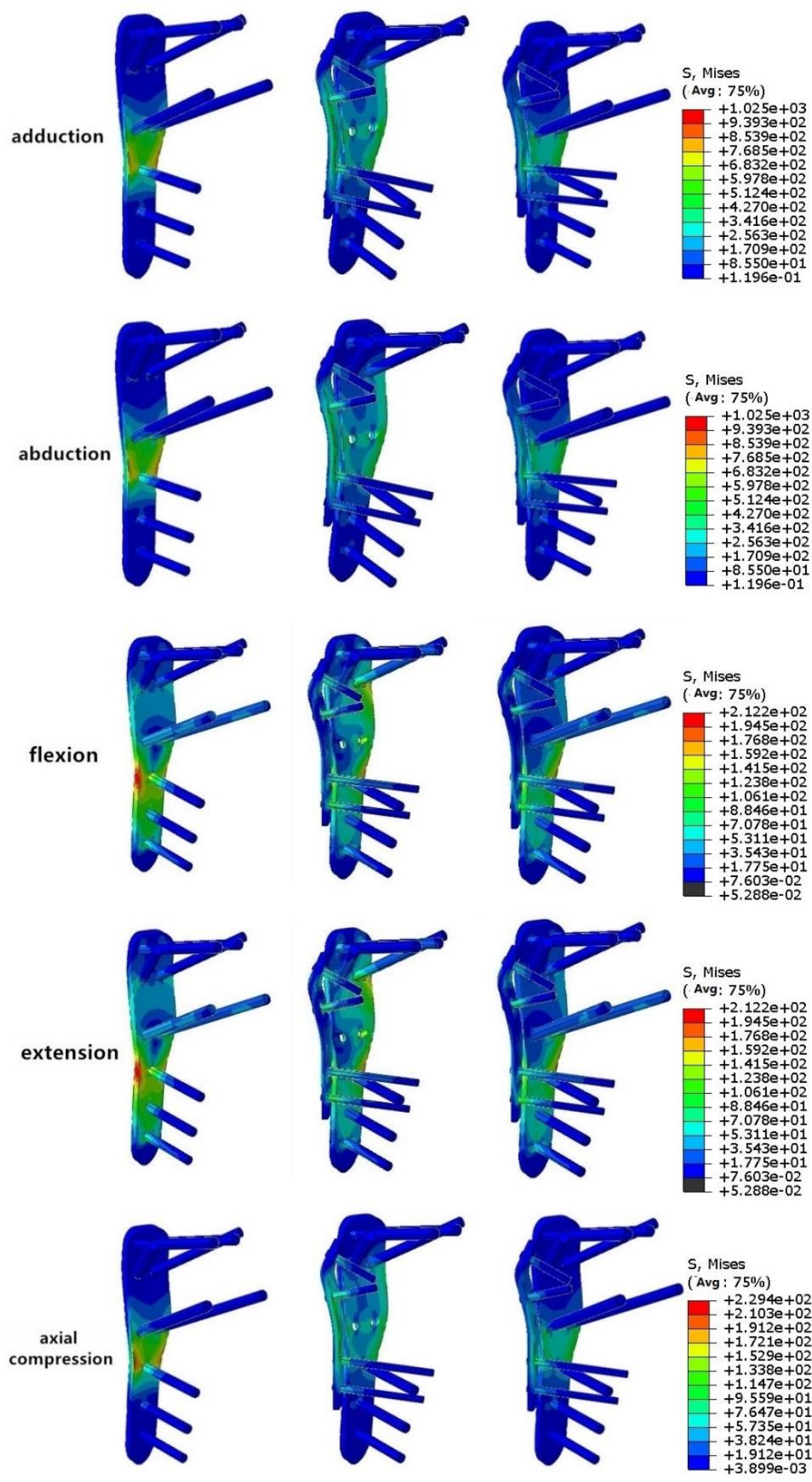

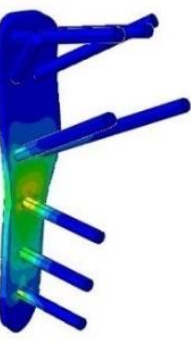

A

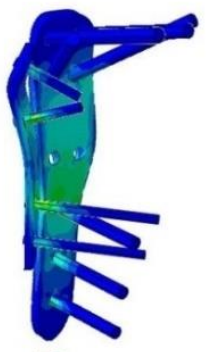

B

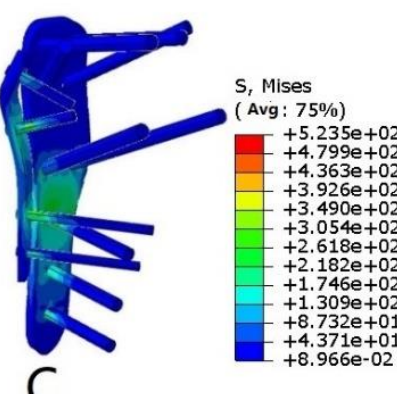

C 


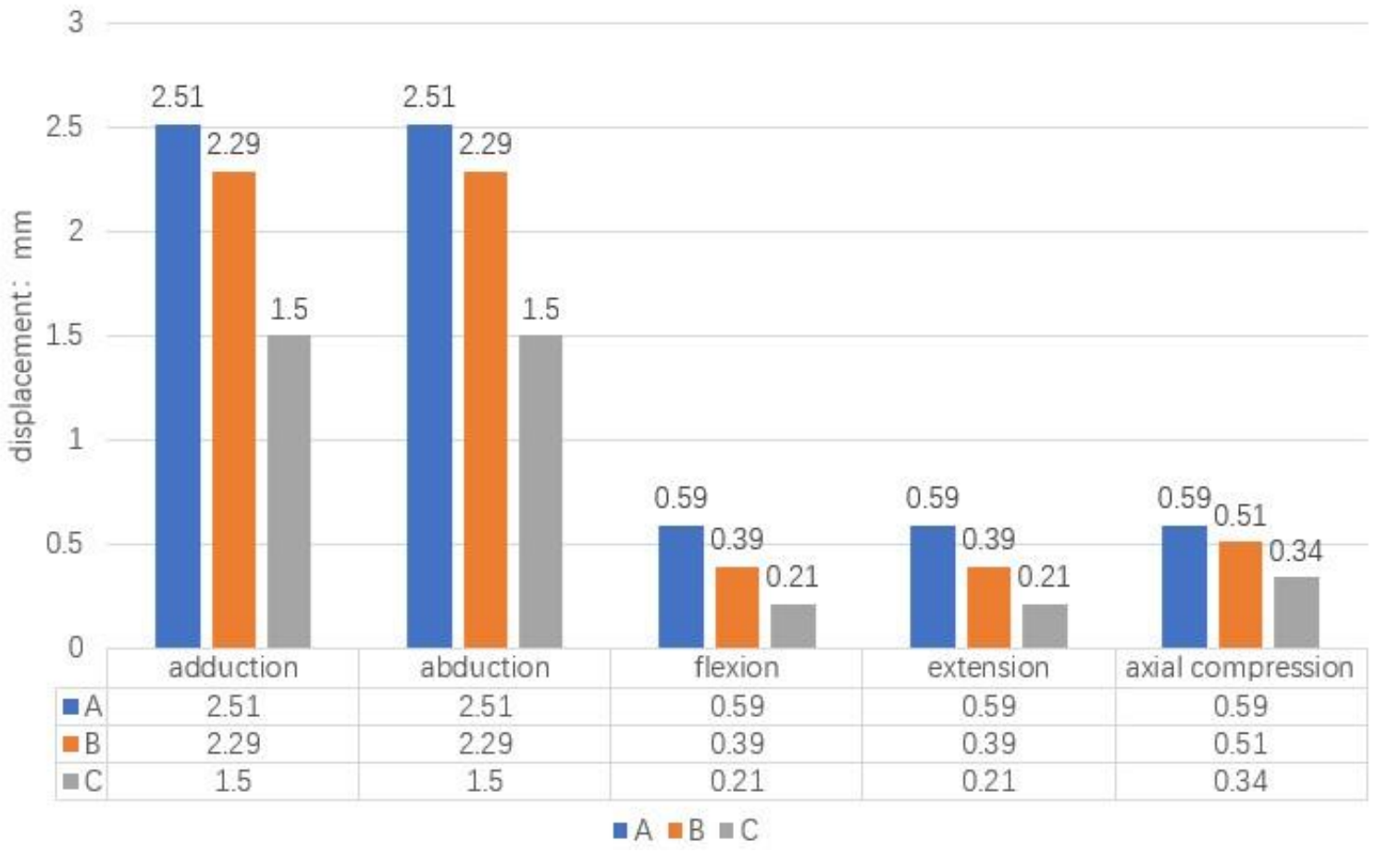

Figure 7. Preoperative imaging findings of a comminuted fracture of the proximal right humerus.

(a): Preoperative $\mathrm{X}$-ray findings. (b, c): Preoperative computed tomography findings.
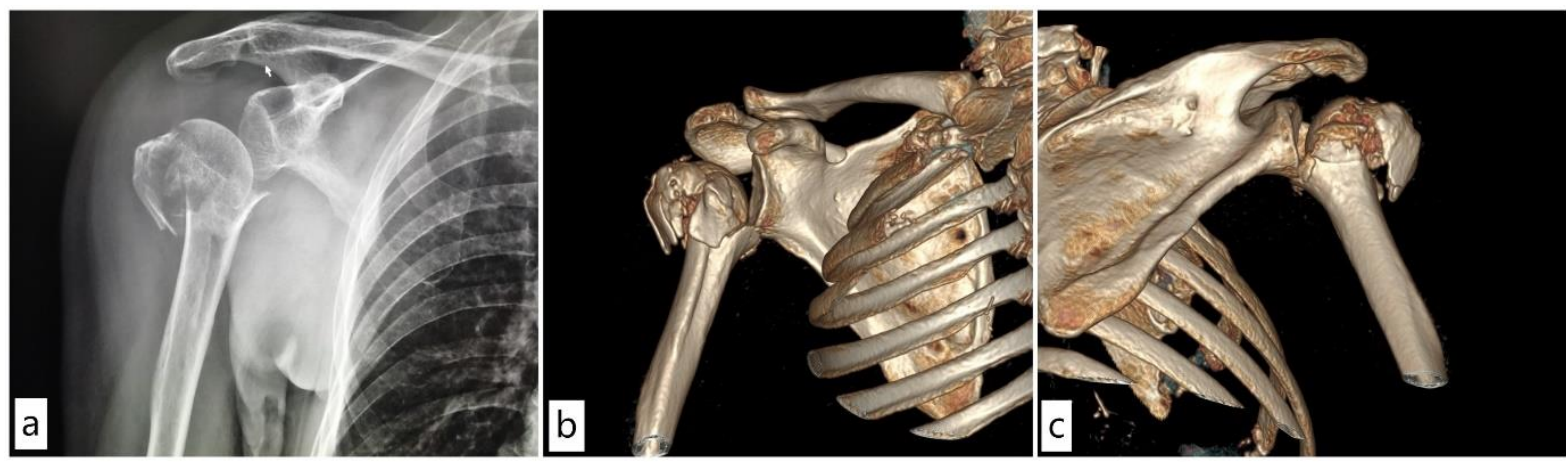

Figure 8. Intraoperative and postoperative images of a comminuted fracture of the proximal

right humerus. (a): Intraoperative use of auxiliary plate reduction fluoroscopy. (b): Intraoperative plate 

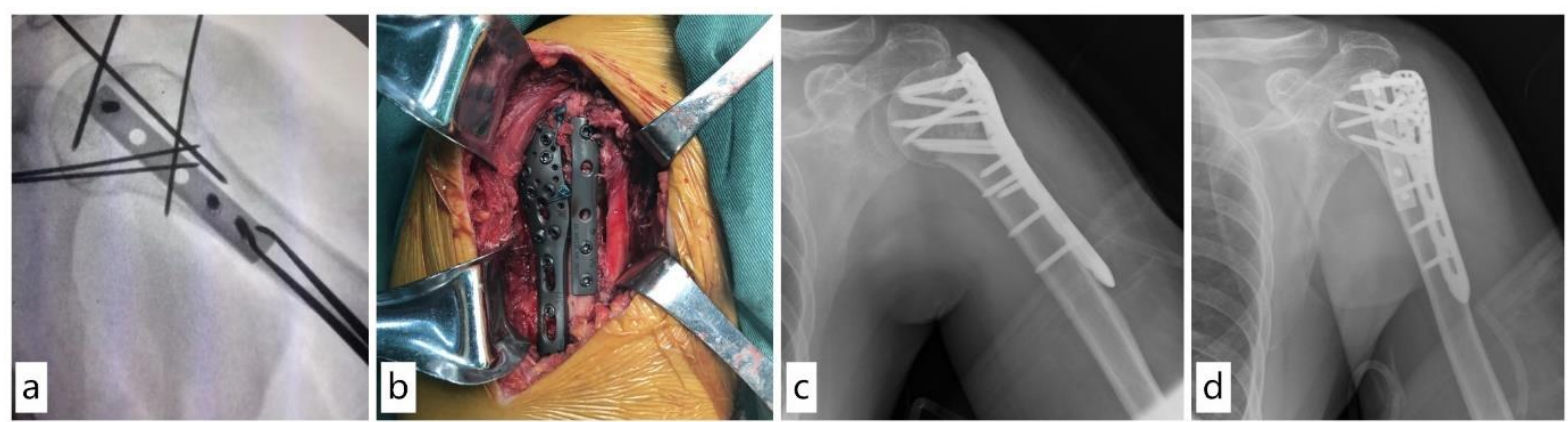

Figure 9. Three-month follow-up results for a comminuted fracture of the proximal right

humerus. (a): X-ray taken 3 months postoperatively showing a healed fracture

with good

positioning of the internal fixation. (b, c): Functional activity at 3 months postoperatively.
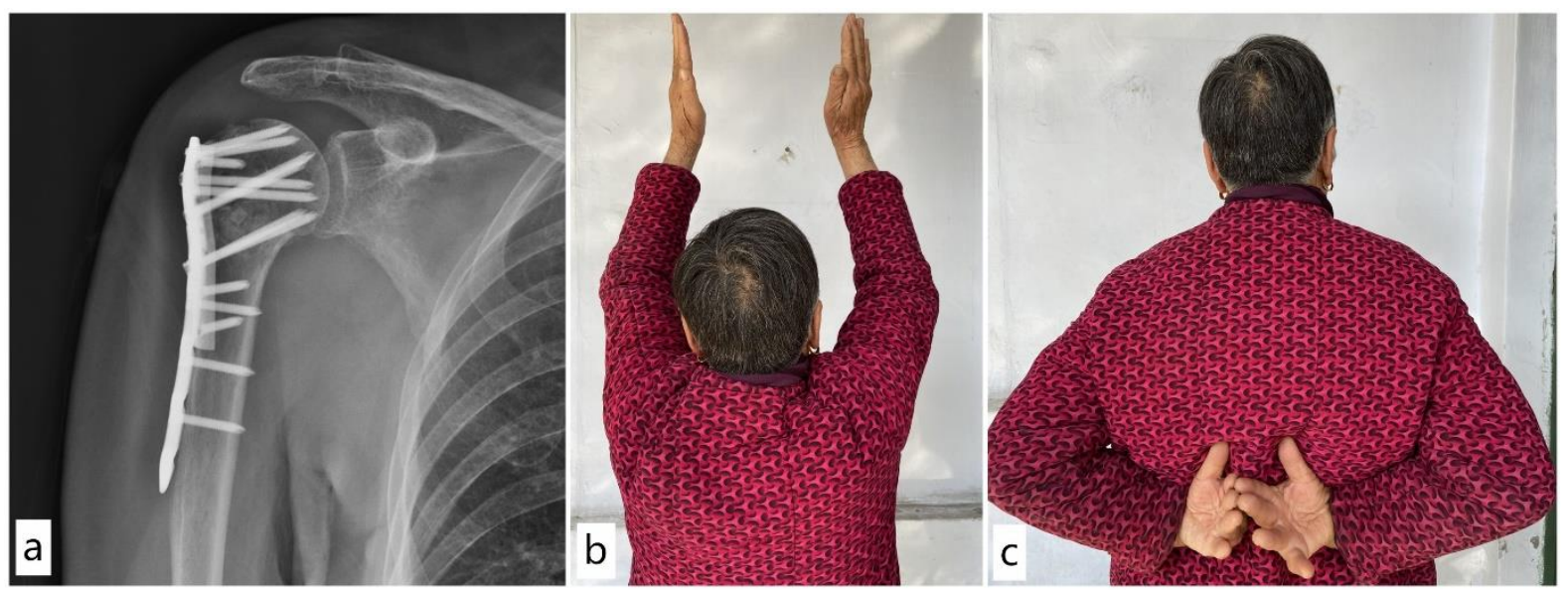
Figures

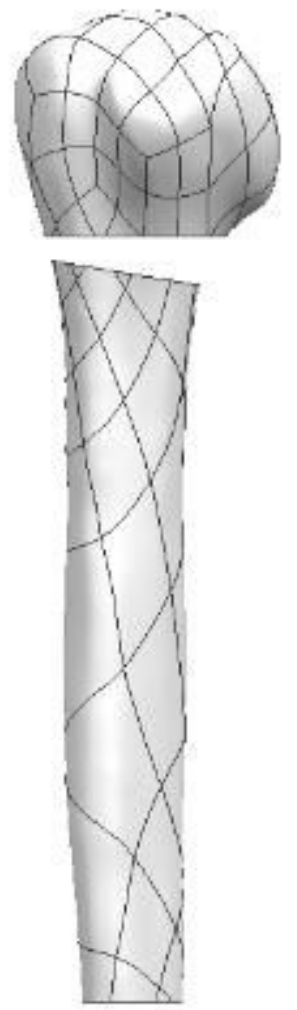

\section{Figure 1}

Bone defect area. A bone defect area with a width of $5 \mathrm{~mm}$ on the lateral side and $10 \mathrm{~mm}$ on the medial side was set at the surgical neck of the humerus to simulate a proximal humeral fracture with an unstable medial column.
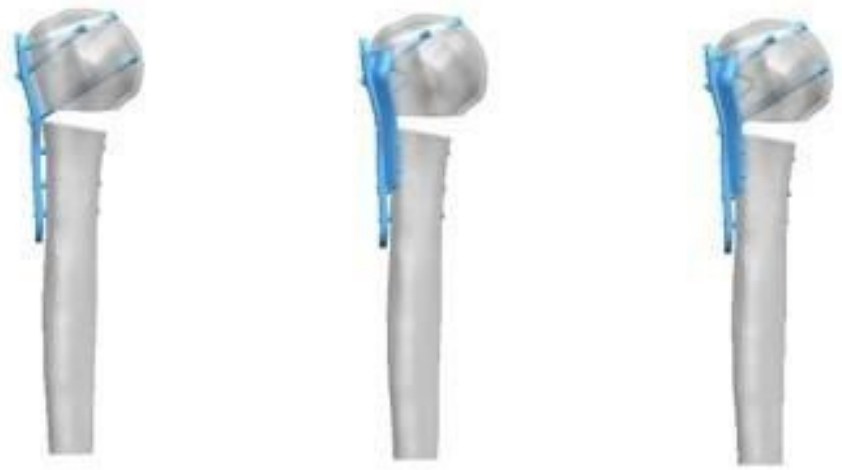

A

B

C

Figure 2 
Three implant models. A: PHILOS plate (PLP). B: PHILOS plate lacking medial screw support and lateral intertubercular sulcus plate (MPLP LSP). C: PHILOS plate and lateral intertubercular sulcus plate (PLP LSP).

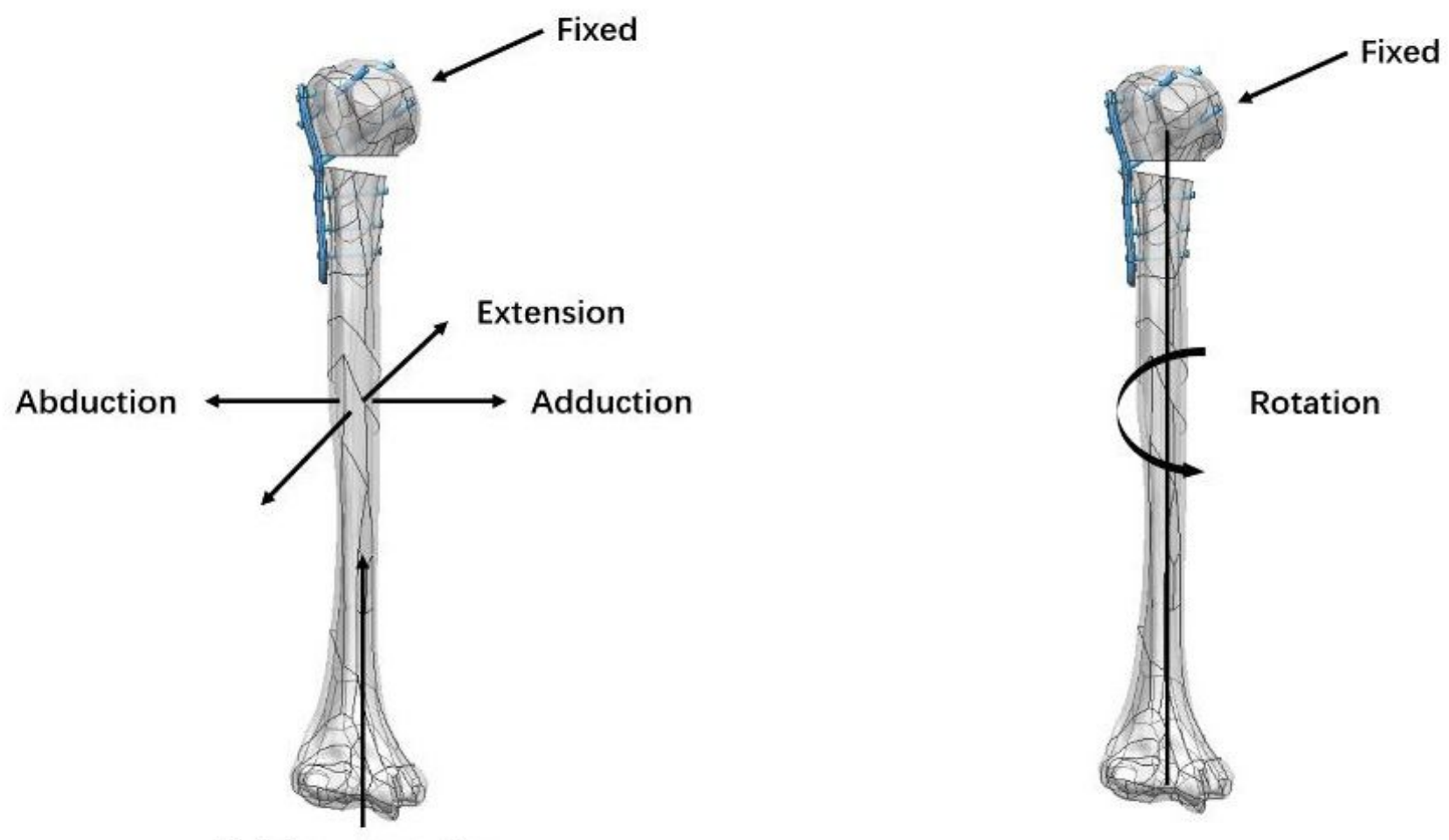

Axial compression

\section{Figure 3}

Load application. Compressive and rotational loads were applied to the humerus model to simulate the functions of the shoulder joint, including abduction, adduction, flexion, extension, axial compression, and internal and external rotation. 

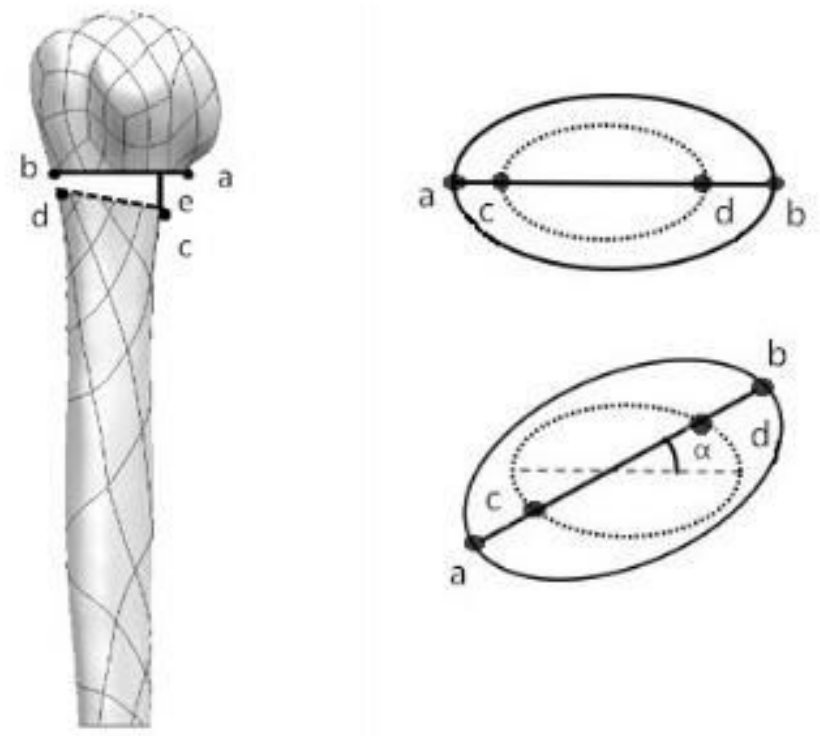

Figure 4

The stability of the fracture region under horizontal and compressive loads was assessed based on the distance covered by the medial fracture gap (line e). The angular variation between the proximal and distal fracture gap was determined to assess regional rotational stability (angle a 


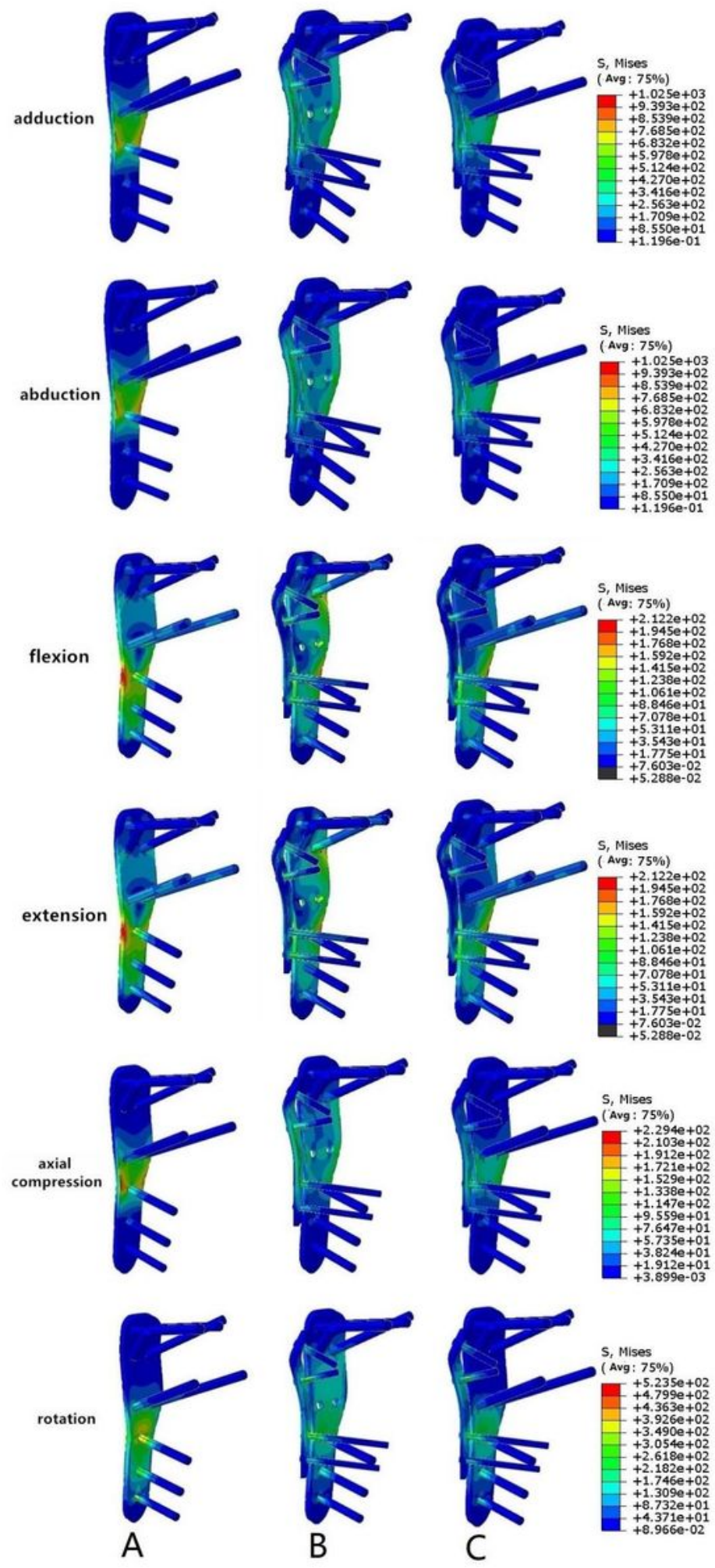

Figure 5

The maximum von Misses stress and stress distribution. Group A (PLP): Full screw PHILOS plate; Group B (MPLP LSP): PHILOS plate without medial screw support plus auxiliary plate; Group C (PLP LSP): full screw PHILOS plate plus auxiliary plate. 


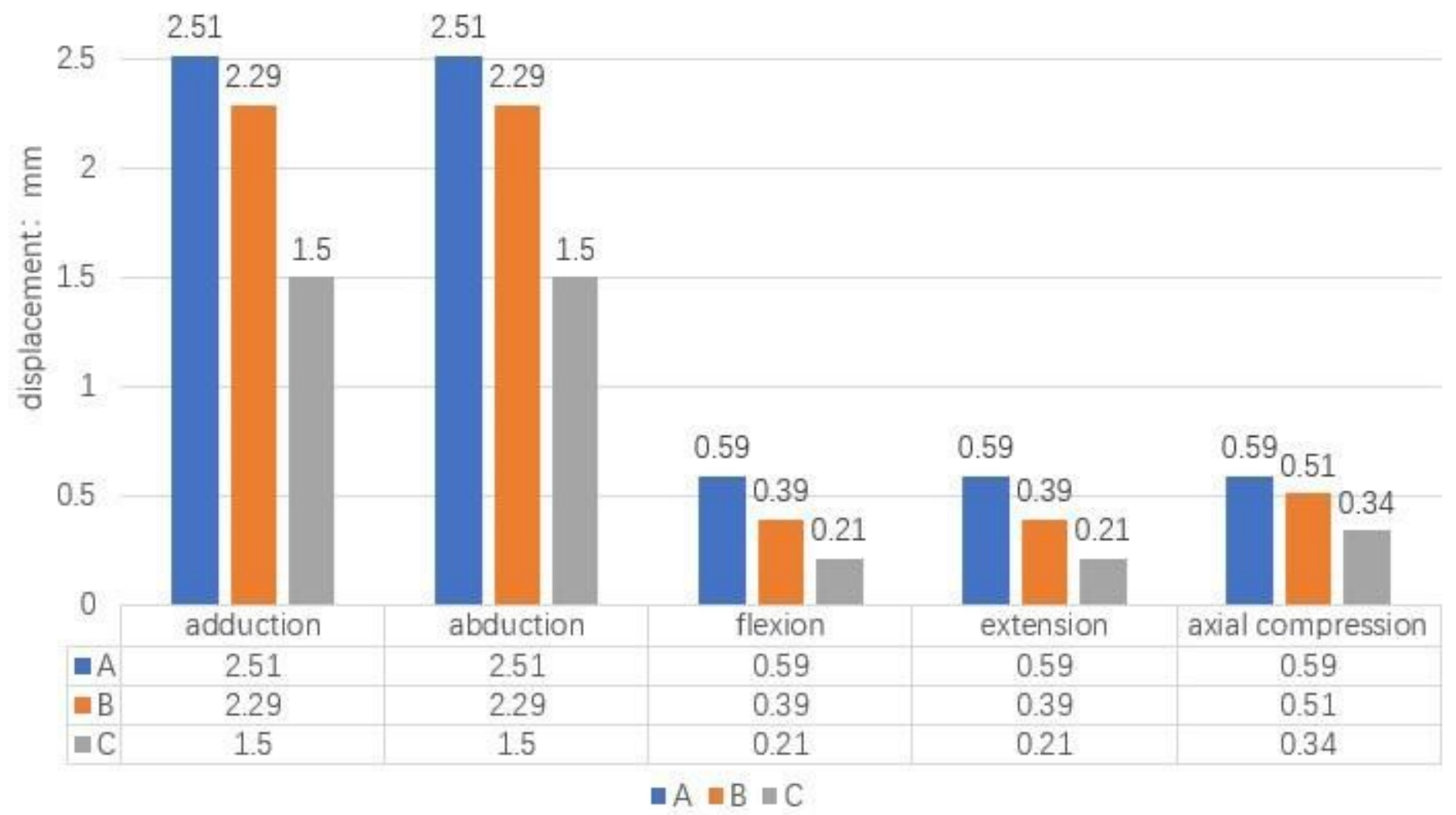

Figure 6

Changes in the displacement of the fracture region under different loading conditions. Group A (PLP): Full screw PHILOS plate; Group B (MPLP LSP): PHILOS plate without medial screw support plus auxiliary plate; Group C (PLP LSP): full screw PHILOS plate plus a uxiliary plate.

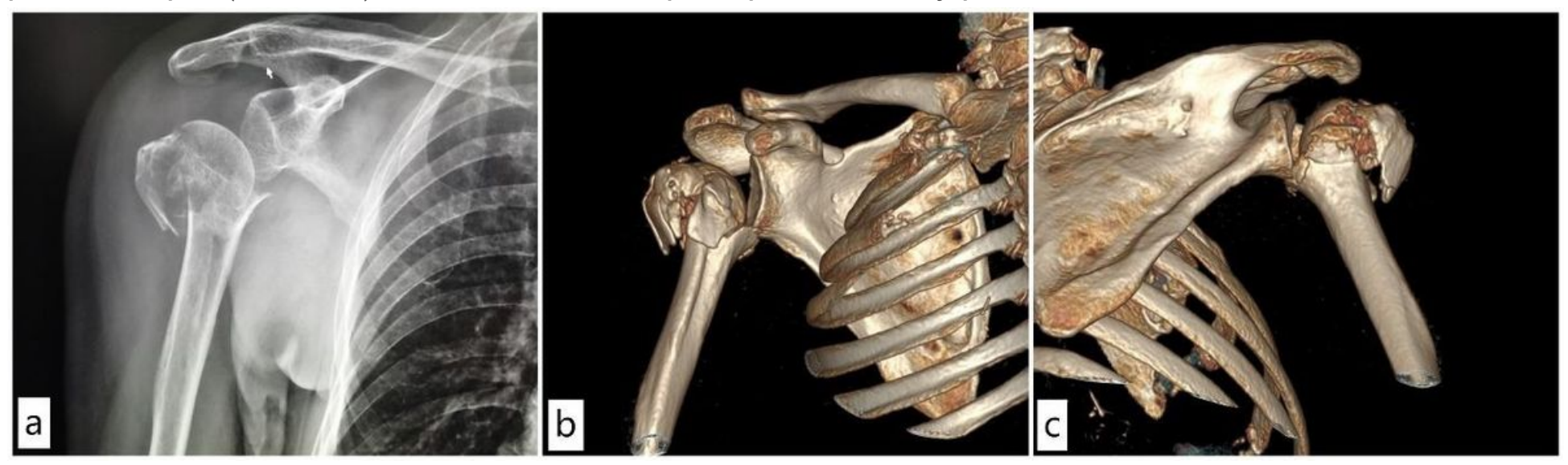

\section{Figure 7}

Preoperative imaging findings of a comminuted fracture of the proximal right humerus. (a ): Preoperative $\mathrm{X}$ ray findings $. \mathrm{b}, \mathrm{c}$ ): Preoperative computed tomography findings 

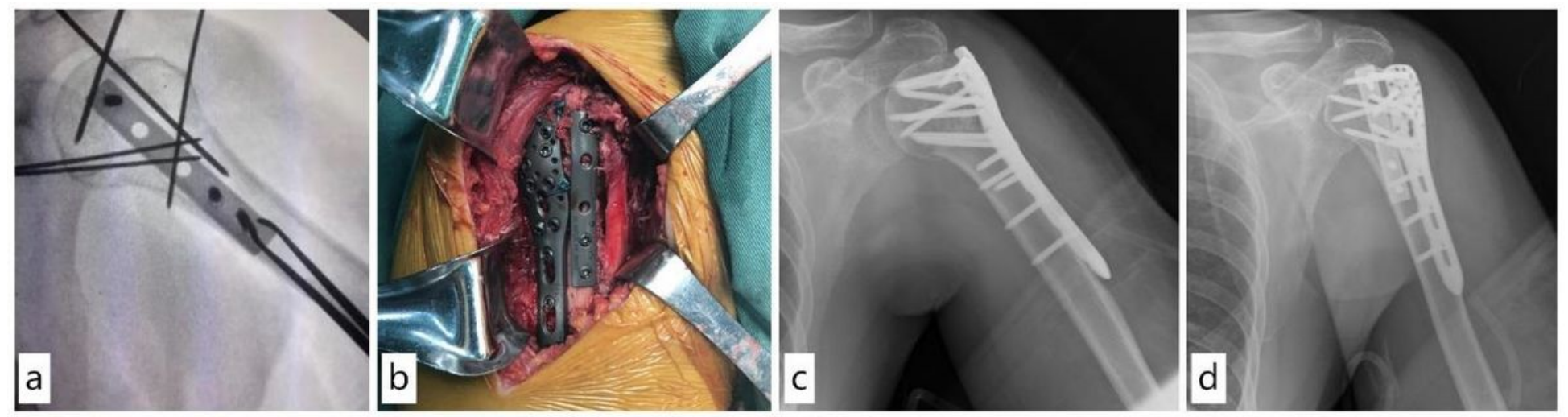

\section{Figure 8}

Intraoperative and postoperative images of a comminuted fracture of the proximal right humerus a ): Intraoperative use of auxiliary plate reduction fluoroscopy. b ): Intraoperative plate placement. (c, d ): Postoperative $\mathrm{X}$ ray positive and lateral radio graphs.
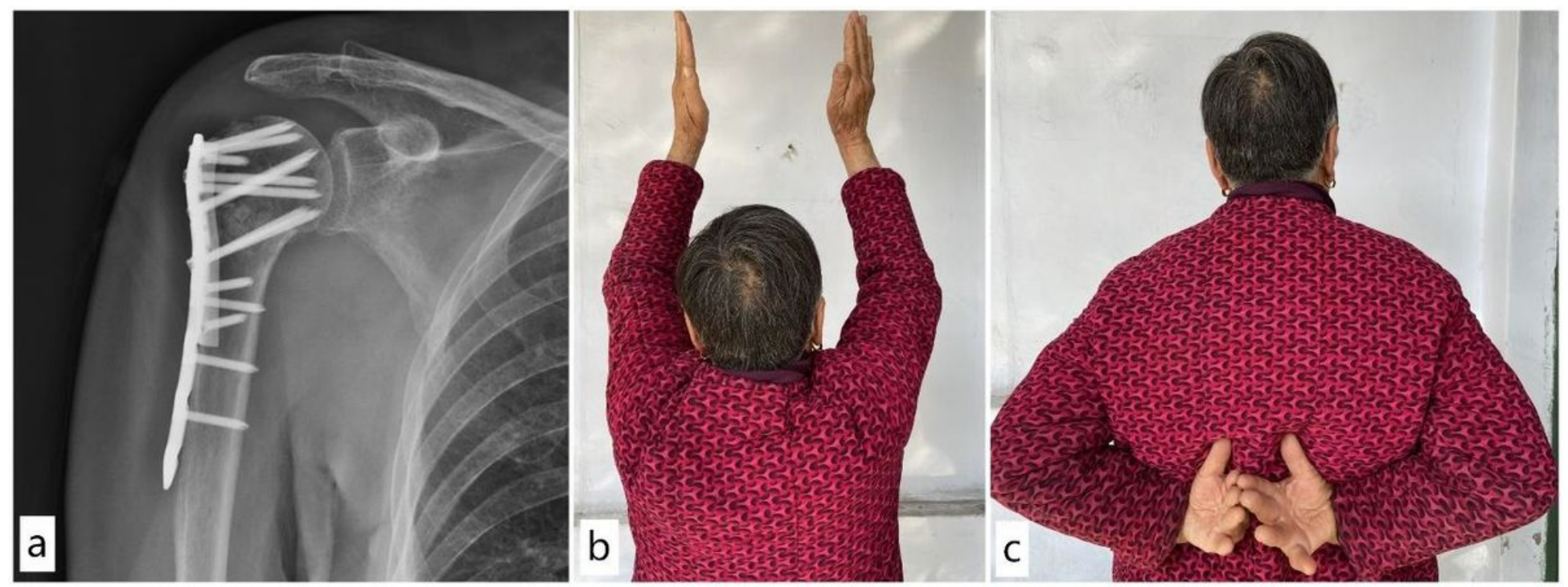

\section{Figure 9}

Three month follow up results for a comminuted fracture of the proximal right humerus . a ): $\mathrm{X}$ ray taken 3 months postoperatively showing a healed fracture with good positioning of the internal fixation. ( $b, c)$ : Functional activity at 3 months postoperatively 